\title{
Microarray analysis of aberrant microRNA expression patterns in spinal cord gliomas of different grades
}

\author{
TIAN AN $^{1 *}$, XIN-QING ZHANG ${ }^{2 *}$, YU-FEI LIU ${ }^{3}$, JUAN LIAN $^{1}$, YAN-XIANG WU ${ }^{1}$, \\ BO-HAN LV ${ }^{1}$, CONG LIANG ${ }^{4}$, CHUN-YOU CHEN ${ }^{5}$, QI-SHUAI YU ${ }^{4}$, \\ MENG-HUA MA ${ }^{5}$, YIN-QIAN WANG ${ }^{2}$, GUANG-JIAN JIANG ${ }^{1}$ and TAO FAN ${ }^{4}$ \\ ${ }^{1}$ School of Traditional Chinese Medicine, Beijing University of Chinese Medicine, Beijing 100029; \\ ${ }^{2}$ Department of Neurosurgery, ChuiYangLiu Hospital Affiliated to Tsinghua University, Beijing 100022; \\ ${ }^{3}$ Beijing University of Chinese Medicine Third Affiliated Hospital, Beijing 100029; \\ ${ }^{4}$ Department of Neurosurgery, Sanbo Brain Hospital, Capital Medical University, Beijing 100093; \\ ${ }^{5}$ Department of Endocrinology, Workers Hospital of Tangshan City, Tangshan, Hebei 063000, P.R. China
}

Received November 19, 2018; Accepted November 15, 2019

DOI: $10.3892 / \mathrm{ol} .2020 .12234$

\begin{abstract}
MicroRNAs (miRNAs) are involved in the development of several types of tumor; however, their role in spinal gliomas remains unknown. The present study aimed to identify potentially novel spinal cord gliomas (SCG)-associated miRNAs and to characterize their roles in the development and progression of SCG. miRNA expression levels in low-grade SCG (classed as stage I-II SCG based on the World Health Organization grading system), high-grade SCG (classed as stage IV SCG based on the World Health Organization grading system) and 5 control cases were measured using a miRNA expression microarray. Subsequently, blood samples from the spinal cord of patients with differing grades of SCG were screened for differentially expressed miRNAs (DEmiRNAs). Compared with the control group, 7 upregulated and 36 downregulated miRNAs were identified in the low-grade SCG group
\end{abstract}

Correspondence to: Professor Guang-Jian Jiang, School of Traditional Chinese Medicine, Beijing University of Chinese Medicine, 11 Bei San Huan Dong Lu, Beijing 100029, P.R. China

E-mail: guangjianjiang@sina.com

Dr Tao Fan, Department of Neurosurgery, Sanbo Brain Hospital, Capital Medical University, 50 Yikesong Road, Beijing 100093, P.R. China

E-mail: fant@ccmu.edu.cn

${ }^{*}$ Contributed equally

Abbreviations: miRNA, microRNA; SCG, spinal cord gliomas; GBM, glioblastoma; DEmiRNAs, differentially expressed miRNAs; DEgenes, differentially expressed genes; GO, gene ontology; BP, biological processes; KEGG, Kyoto Encyclopedia of Genes and Genomes; CDK6, cyclin-dependent kinase 6; FC, Fold change

Key words: spinal cord gliomas, circulating microRNA, biomarkers, miR-1246 and a total of 70 upregulated and 20 downregulated miRNAs were identified in the high-grade SCG group $(\mathrm{P} \leq 0.05$, fold change $>2$ ). Gene Ontology analysis revealed that the regulation of cellular metabolic processes, negative regulation of biological processes and axon guidance were primarily involved. Moreover, pathway analysis showed that the target genes of DEmiRNAs were enriched in tumor-related signaling pathways, such as the MAPK and Wnt signaling pathway. The results suggest that DEmiRNAs in peripheral blood may serve as novel target markers with high specificity and sensitivity for the diagnosis of SCG.

\section{Introduction}

The World Health Organization (WHO) classifies human spinal cord gliomas (SCGs) into four different grades (I-IV), according to the increase in malignancy (1). Low-grade SCGs (classed as WHO stage I-II) have a tendency for local recurrence and may trend towards the development of high-grade SCG (classed as WHO stage IV) (2). Post-surgery, patients with low-grade SCG usually exhibit recurrence of tumors within months or years, whereas patients with high-grade SCG, also known as glioblastoma, have a median survival period of only 1 year $(3,4)$. Therefore, studies exploring the mechanism underlying occurrence and progression of SCG may assist in improving our understanding of the biological behavior of SCG and thus result in improved treatment strategies.

MicroRNAs (miRNAs/miRs) are a class of endogenous non-coding small RNAs ( 22 nucleotides in length) which negatively regulate target genes at the post-transcriptional level by incomplete binding to the target gene $(5,6)$. miRNAs, which are hypothesized to serve as targets for tumor therapy, can promote cell proliferation, inhibit apoptosis, promote angiogenesis and serve a regulatory role in tumor pathogenesis (7-9). Furthermore, miRNAs may be involved in tumor resistance and may be a valuable diagnostic and prognostic factor for patients with SCG (10). 
To the best of our knowledge, the expression of miRNAs in SCG has not been extensively studied, particularly regarding miRNAs which may be implicated in the development of SCG. The present study used miRNA microarrays to detect differentially expressed (DE)miRNAs in the blood of patients with different grades of SCG. The identification of novel DEmiRNAs may provide novel insights into the progression and treatment of SCG.

\section{Materials and methods}

Study patients and blood samples. The present study was approved and supervised by The Ethics Committees of Beijing University of Chinese Medicine (Beijing, China) and Sanbo Brain Hospital, Capital Medical University (Beijing, China). Written informed consent was obtained from all patients prior to the study start. According to the WHO (2016) (1) classification of neurological tumors, a total of 10 patients with SCG who underwent surgical treatment at Sanbo Brain Hospital, without preoperative chemotherapy or radiotherapy were recruited between February 2016 to December 2016, and blood samples were collected from low-grade or high-grade SCG cases. In addition, blood was collected from five patients with benign tumors as a control. Arterial blood was stored in liquid nitrogen immediately after extraction.

Microarray determination. Total miRNAs were labeled and hybridized according to the manufacturers' protocol (Qiagen China Co., Ltd.). The slides were scanned using the Axon GenePix 4000B microarray scanner (Molecular Devices, LLC.). The scanned images were imported into GenePix Pro version 6.0 (Molecular Devices, LLC.) for grid alignment and data extraction. After median normalization, significant DEmiRNAs between the two groups were identified based on the fold change (FC) values and P-value.

Reverse transcription quantitative $(R T-q) P C R$. Total RNA was extracted as previously described (11). Total RNA was extracted from the blood of patients with SCG and control patients using TRIzol ${ }^{\circledR}$ reagent (Invitrogen; Thermo Fisher Scientific Inc.). The RNA quantity and quality were determined using a NanoDrop spectrophotometer (ND-1000, NanoDrop Technologies; Thermo Fisher Scientific, Inc.). Total RNA was reverse transcribed into cDNA using the cDNA Reverse Transcription kit (Invitrogen; Thermo Fisher Scientific Inc.), with $2 \mu \mathrm{g}$ total RNA. qPCR was subsequently performed using SYBR Premix ExTaq and the MX3000 instrument (Kangchen BioTech Co., Ltd.), according to the manufacturer's protocol. RT-qPCR was performed using a 7500 Real-Time PCR System (Applied Biosystems; Thermo Fisher Scientific, Inc.), accordingly to the manufacturer's protocol. The sequences of the primers used are presented in Table I. mRNA expression levels were quantified using the $22^{-\Delta \Delta \mathrm{Cq}}$ method (11) and normalized to the internal reference gene U6.

Differential miRNA target gene prediction. MirDB (version 5; http://www.mirdb.org) and TargetScan (version 7.1; http://www.targetscan.org) databases were used to predict and analyze the target genes of the abnormally expressed miRNAs.
The final results from the prediction utilized the overlapping regions of the gene obtained between the two databases.

Gene Ontology (GO) and Kyoto Encyclopedia of Genes and Genomes (KEGG) pathway analyses. The GO database (http://geneontology.org) includes molecular function, cellular component and biological processes as the three categories to stratify function. GO analysis was used to obtain the significantly enriched GO articles and corresponding genes in order to deduce the biological functions of DEmiRNAs in SCG.

Using the KEGG database (https://www.kegg.jp), the pathways most associated with the DEmiRNAs were determined.

Statistical analysis. SPSS software (version 20.0; IBM, Corp.) was used for statistical analysis. The results are expressed as the mean \pm standard error of the mean. Unpaired Student's t-test was used to calculate the P-value between the normalized signals of the two sets of samples and the standardized signal values were used to calculate the $\mathrm{FC}(\mathrm{FC}>2, \mathrm{P}<0.05)$. A Fisher's exact test was used to identify which pathways and GO entries were significantly associated with the DEmiRNAs. $\mathrm{P}<0.05$ was considered to indicate a statistically significant difference.

\section{Results}

Patient characteristics. A total of 10 patients with SCG, consisting of five patients with high-grade SCG and five patients with low-grade SCG were recruited. Blood samples were collected and staged according to the WHO (2016) classification of neurological tumors. Basic information on the patients, including age, sex and tumor location are presented in Table II.

DEmiRNAs in the blood of patients with SCG. Compared with the normal group, there were 156 upregulated miRNAs and 84 downregulated miRNAs $(\mathrm{FC}>2)$ in the low-grade SCG group (Table SI). Among these, seven upregulated miRNAs and 36 downregulated miRNAs were statistically significant $(\mathrm{P}<0.05$; Tables III and IV). In the high-grade group, 251 upregulated miRNAs and 35 downregulated miRNAs were identified ( $\mathrm{FC}>2)$. Of these, 70 upregulated and 20 downregulated miRNAs were statistically significant $(\mathrm{P}<0.05, \mathrm{FC}>2$; Table SII). The number of DEmiRNAs in the high-grade group was higher compared with the low-grade SCG group, in terms of the expression profiles of miRNAs in the blood of the tumor patients (Fig. 1A). The top 10 most upregulated and downregulated genes are listed in Tables $\mathrm{V}$ and VI.

To validate the results of the microarray analysis, two DEmiRNAs were randomly chosen for RT-qPCR; miR-3189-3p was upregulated in both the low-grade and the high-grade group and miR-4527 was upregulated in the high-grade group. The results obtained from RT-qPCR were consistent with those of the microarray analysis and showed statistical significance ( $\mathrm{P}<0.05$; Fig. 2). All of the data on dysregulated expression of the DEmiRNAs identified are presented in Tables SI and SII.

Target prediction for the DEmiRNAs in the low-grade and high-grade groups. The top 18 and 20 miRNAs in the 
Table I. miRNA and mRNA primers for quantitative PCR analysis.

\begin{tabular}{ll}
\hline Primer & \multicolumn{1}{c}{ Sequence } \\
\hline U6 & \\
Forward & 5'-GCTTCGGCAGCACATATACTAAAAT-3' \\
Reverse & 5'-CGCTTCACGAATTTGCGTGTCAT-3' \\
hsa-miR-3189-3p & \\
Forward & 5'-CCGCGCCCTTGGGTCTGATG-3' \\
Reverse & 5'-ATCCAGTGCAGGGTCCGAGG-3' \\
miRNA specific primer & GTCGTATCCAGTGCAGGGTCCGAGGTATTCGCACTGGATACGACCTACCC \\
hsa-miR-4527 & \\
Forward & 5'-AGCCGCGTGGTCTGCAAACACAT-3' \\
Reverse & 5'-ATCCAGTGCAGGGTCCGAGG-3' \\
miRNA specific primer & GTCGTATCCGCAGGGTCCGAGGTATTCGCACTGGATACGACACAGTC \\
\hline miR, microRNA. & \\
\hline
\end{tabular}

Table II. Clinical characteristics of the patients.

\begin{tabular}{|c|c|c|c|c|c|}
\hline Patient no. & Age, years & Sex & Tumor pathology classification & Tumor grade & Tumor location \\
\hline 25343 & 28 & Female & Glioblastoma, NOS & IV & T10-L1 \\
\hline 26600 & 15 & Male & Glioblastoma, NOS & IV & $\mathrm{C} 5-7$ \\
\hline $26600(2)$ & 17 & Male & Glioblastoma, NOS & IV & $\mathrm{T} 1-5$ \\
\hline 28553 & 14 & Female & Glioblastoma, NOS & IV & $\mathrm{T} 1-8$ \\
\hline 28378 & 11 & Male & Glioblastoma, NOS & IV & T9-12 \\
\hline 26051 & 48 & Male & Astrocytoma, NOS & II & $\mathrm{C} 1-3$ \\
\hline 506353 & 47 & Female & Astrocytoma, NOS & II & T5-7 \\
\hline 511691 & 45 & Female & Astrocytoma, NOS & II & C6-T1 \\
\hline 27110 & 17 & Male & Hair cell astrocytoma & I & $\mathrm{T} 1-2$ \\
\hline 503550 & 13 & Male & Hair cell astrocytoma & I & T1-4 \\
\hline 1 & 38 & Female & Arnold-chiari deformity & Control & Craniotomy \\
\hline 2 & 36 & Male & Atlantoaxial dislocation & Control & C1-3 \\
\hline 3 & 25 & Female & Atlantoaxial dislocation & Control & C1-3 \\
\hline 4 & 19 & Female & Hemifacial spasm & Control & Craniotomy \\
\hline 5 & 13 & Male & Trauma & Control & $\mathrm{T} 1-3$ \\
\hline
\end{tabular}

C, cervical; L, lumbar; T, thoracic; NOS, not otherwise specified.

Table III. Upregulated miRNAs in the blood of patients with low grade spinal cord glioma.

\begin{tabular}{llrr}
\hline Patient ID & \multicolumn{1}{c}{ miRNA } & Fold change & P-value \\
\hline 168870 & hsa-miR-1246 & 2.1296 & $0.0002^{\mathrm{c}}$ \\
46808 & hsa-miR-4485-3p & 14.7751 & $0.0013^{\mathrm{b}}$ \\
168558 & hsa-miR-5585-3p & 2.5819 & $0.0072^{\mathrm{b}}$ \\
168568 & hsa-miR-1290 & 2.1757 & $0.0078^{\mathrm{b}}$ \\
147701 & hsa-miR-491-3p & 3.1754 & $0.0111^{\mathrm{a}}$ \\
46221 & hsa-miR-519d-3p & 2.1241 & $0.0304^{\mathrm{a}}$ \\
148377 & hsa-miR-3653-3p & 2.2735 & $0.0446^{\mathrm{a}}$ \\
\hline
\end{tabular}

${ }^{\mathrm{a}} \mathrm{P}<0.05,{ }^{\mathrm{b}} \mathrm{P}<0.01,{ }^{\mathrm{c}} \mathrm{P}<0.001$. miR, microRNA. 
Table IV. Top 10 downregulated miRNAs in blood of patients with low grade spinal cord glioma.

\begin{tabular}{lllr}
\hline Patient ID & \multicolumn{1}{c}{ miRNA } & Fold change & P-value \\
\hline 146159 & hsv1-miR-H4-3p & 0.1415 & 0.0237 \\
147707 & hsa-miR-3154 & 0.1485 & 0.0130 \\
147796 & hsa-miR-4327 & 0.1643 & 0.0375 \\
11004 & hsa-miR-203a-3p & 0.2281 & 0.0138 \\
148278 & hsa-miR-138-2-3p & 0.2355 & 0.0128 \\
146163 & hsa-miR-224-3p & 0.2454 & 0.0375 \\
46567 & hsa-miR-3176 & 0.2456 & 0.0248 \\
147627 & hsa-miR-4294 & 0.2740 & 0.0199 \\
169013 & hsa-miR-5195-5p & 0.2849 & 0.0245 \\
168684 & hsa-miR-5010-5p & 0.2985 & 0.0248
\end{tabular}

${ }^{\mathrm{a} A l l} \mathrm{P}<0.05 . \mathrm{miR}$, microRNA.
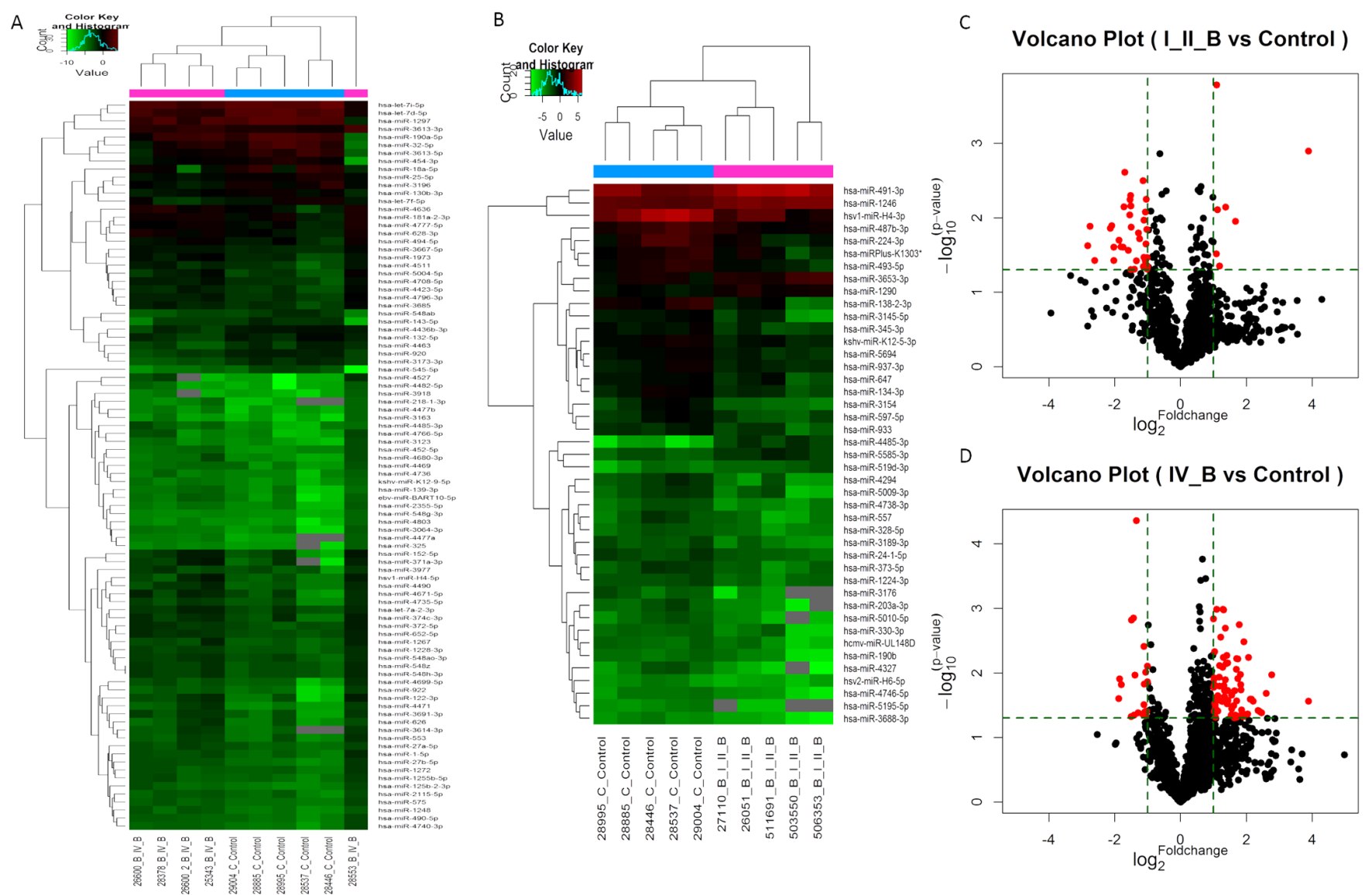

Figure 1. DEmiRNAs in arterial blood between the high-grade and low-grade SCG groups compared with the control group. Hierarchical clustering analysis of the differentially expressed miRNAs in the serum of patients with (A) high-grade or (B) low-grade SCG. FC $>2 ; \mathrm{P}<0.05 ; \mathrm{n}=5$. Expression values are expressed in different colors, red for upregulated expression and green for downregulated expression. Volcano plots constructed using the FC and P-values for patients with (C) low-grade and (D) high-grade SCG. The vertical line corresponds to a 2-fold up or downregulation of expression in the serum of both groups and the horizontal line represents the P-value. Red points represent DEmiRNAs with statistical significance whereas black points represent DEmiRNAs with no statistical significance. DEmiRNAs, differentially expressed microRNAs; SCG, spinal cord glioma; FC, fold change; I_II_B, low grade SCG; IV_B SCG, high grade.

low-grade and high-grade groups, respectively, were selected for prediction of their target genes, based on the FC values for the DEmiRNAs. A total of 348 and 728 target genes for the upregulated and downregulated miRNAs, respectively in the low-grade group were identified (Table SIII).
In addition, 772 and 1,731 target genes were predicted for the upregulated and downregulated miRNAs, respectively, in the high-grade group (Table SIV). The network diagram of the 4 downregulated miRNAs associated with spinal glioma development, and their target genes from the 
Table V. Top 10 upregulated miRNAs in blood of patients with high grade glioma.

\begin{tabular}{llcr}
\hline Patient ID & \multicolumn{1}{c}{ miRNA } & Fold change & P-value $^{\mathrm{a}}$ \\
\hline 169006 & hsa-miR-4527 & 14.7266 & $0.0274^{\mathrm{a}}$ \\
169029 & hsa-miR-4471 & 6.8163 & $0.0107^{\mathrm{a}}$ \\
42551 & hsa-miR-122-3p & 6.0684 & $0.0207^{\mathrm{a}}$ \\
169249 & hsa-miR-4482-5p & 5.4977 & $0.0413^{\mathrm{a}}$ \\
42941 & hsa-miR-218-1-3p & 5.3011 & $0.0396^{\mathrm{a}}$ \\
168860 & hsa-miR-4766-5p & 5.1045 & $0.0381^{\text {a }}$ \\
146157 & hsv1-miR-H4-5p & 4.5925 & $0.0274^{\mathrm{a}}$ \\
169223 & hsa-miR-4680-3p & 4.4346 & $0.0256^{\mathrm{a}}$ \\
46808 & hsa-miR-4485-3p & 4.3325 & $0.0254^{\mathrm{a}}$ \\
169369 & hsa-miR-4490 & 4.1875 & $0.0058^{\mathrm{b}}$ \\
\end{tabular}

${ }^{\mathrm{a}} \mathrm{P}<0.05,{ }^{\mathrm{b}} \mathrm{P}<0.001 . \mathrm{miR}$, microRNA.

Table VI. Top 10 downregulated miRNAs in blood of patients with high grade glioma.

\begin{tabular}{lllr}
\hline Patient ID & \multicolumn{1}{c}{ miRNA } & Fold change & P-value $^{\mathrm{a}}$ \\
\hline 147195 & hsa-miR-18a-5p & 0.2722 & $0.0249^{\mathrm{a}}$ \\
42956 & hsa-miR-545-5p & 0.2765 & $0.0123^{\mathrm{a}}$ \\
147334 & hsa-miR-3613-5p & 0.2877 & $0.0152^{\mathrm{a}}$ \\
11053 & hsa-miR-32-5p & 0.3543 & $0.0472^{\mathrm{a}}$ \\
147845 & hsa-miR-3173-3p & 0.3550 & $0.0015^{\mathrm{b}}$ \\
145968 & hsa-let-7d-5p & 0.3723 & $0.0014^{\mathrm{b}}$ \\
147817 & hsa-miR-3196 & 0.3755 & $0.0450^{\mathrm{a}}$ \\
148620 & hsa-miR-454-3p & 0.3825 & $0.0108^{\mathrm{a}}$ \\
42932 & hsa-miR-920 & 0.3947 & $0.0000^{\mathrm{c}}$ \\
17752 & hsa-let-7f-5p & 0.4082 & $0.0415^{\mathrm{a}}$ \\
\hline
\end{tabular}

${ }^{\mathrm{a}} \mathrm{P}<0.05,{ }^{\mathrm{b}} \mathrm{P}<0.01,{ }^{\mathrm{c}} \mathrm{P}<0.001 . \mathrm{miR}$, microRNA.

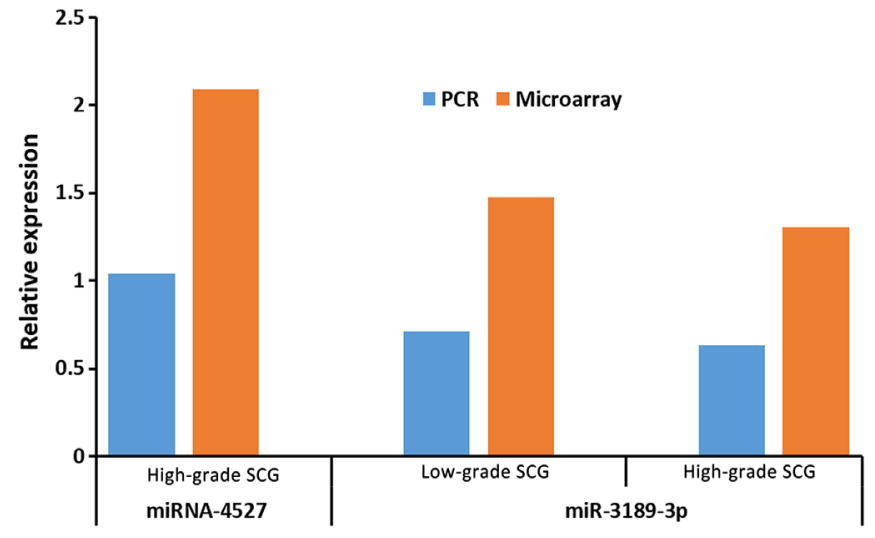

Figure 2. Validation of the microarray data using RT-qPCR. Comparison of the results from RT-qPCR and microarray analysis for miR-3189-3p and miR-4527. The results obtained from these two methods were consistent. $\mathrm{n}=6$. RT-qPCR, reverse transcription quantitative PCR; miR, microRNA; SCG, spinal cord glioma.

low-grade (hsa-miR-3154, hsa-miR-4327, hsa-miR-203a-3p and hsa-miR-138-2-3p) and high-grade (hsa-miR-18a-5p,
hsa-miR-545-5p, hsa-miR-3613-5p and hsa-miR-32-5p) blood sample groups are presented in Figs. 3 and 4, respectively.

GO analysis of the DEmiRNAs in the low-grade and high-grade groups. GO analysis of the differentially expressed genes was performed. The results of the low-grade group showed that the coding genes adjacent to the downregulated miRNAs were primarily involved in the 'negative regulation of biological process' and 'positive regulation of intracellular transport', and the upregulated miRNAs were primarily enriched in 'axon guidance' and 'neuron projection guidance'. In addition, the most enriched GO targets for the high-grade group were 'regulation of cellular metabolic process' (downregulated) and 'axon development' (upregulated) (Figs. 5 and 6; Tables SV and SVI).

Pathway analysis of the DEmiRNAs in the low-grade and high-grade groups. Based on the KEGG database, the significance levels of the differentially expressed gene pathways were analyzed. Results showed that the downregulated miRNAs in the low-grade group were primarily involved in the 'FoxO signaling pathway', and the upregulated genes 


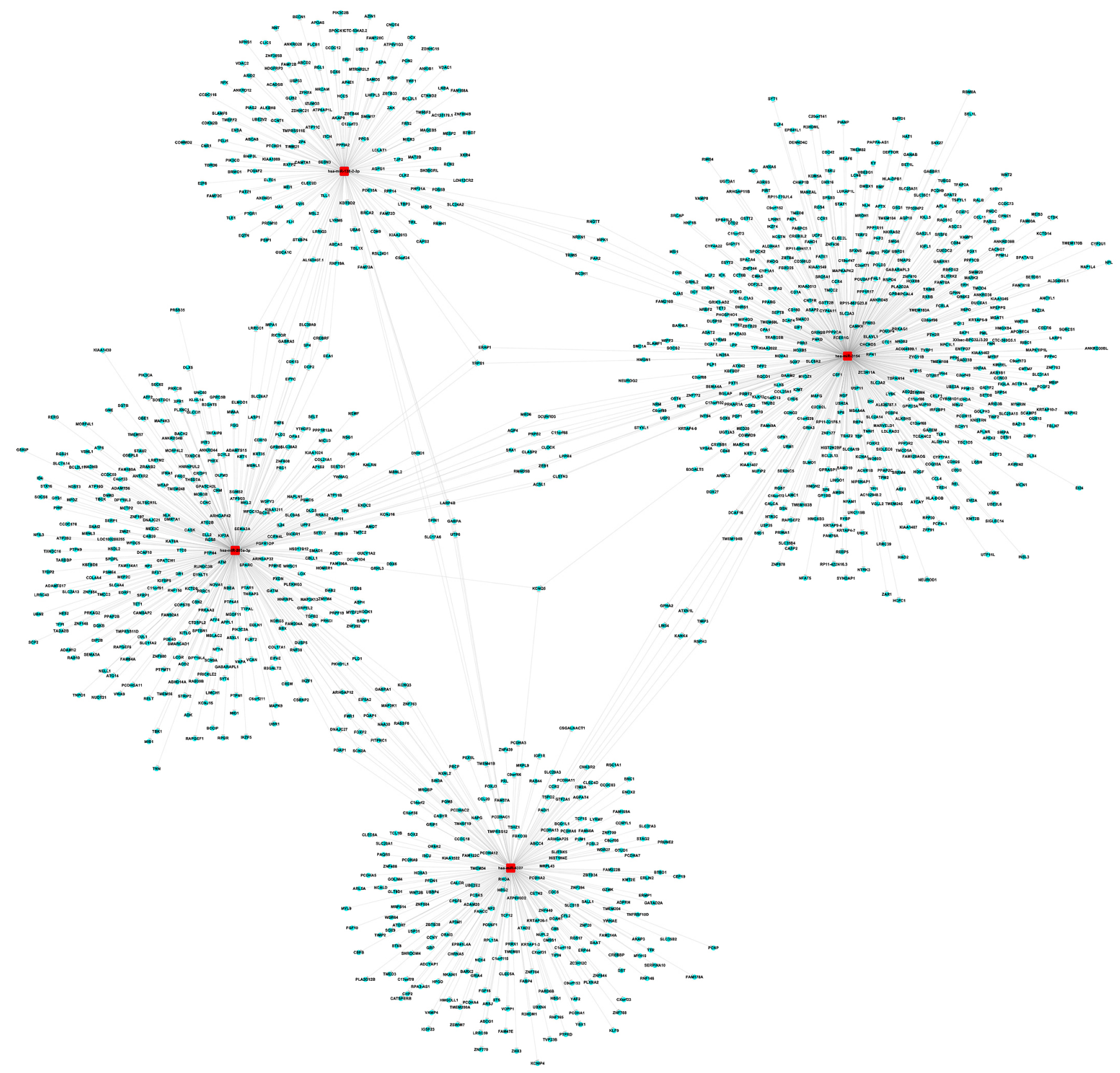

Figure 3. Differentially expressed miRNA-mRNA networks of hsa-miR-3154, hsa-miR-4327, hsa-miR-203a-3p and hsa-miR-138-2-3p in patients with low grade spinal cord glioma. Square red nodes and round blue nodes represent downregulated miRNAs and mRNAs, respectively, and edges between two nodes represents an association. miR, microRNA.

were primarily involved in 'Osteoclast differentiation' and the 'Wnt signaling pathway'. Furthermore, the differentially expressed genes in the high-grade group were primarily involved in the 'circadian rhythm', 'Longevity regulating pathway', 'mTOR signaling pathway' and 'Signaling pathways regulating pluripotency of stem cells' (Fig. 7). The top 10 pathways in which the DEmiRNAs were involved in are listed in descending order of the enrichment factors in Fig. 7.

Similarities between identified DEmiRNAs in the low-grade and high-grade groups. To examine the differences in miRNA expression between the low-grade and high-grade groups, the DEmiRNA data for both groups were compared, and DEmiRNAs that were present in both groups were selected.
A total of 27 upregulated miRNAs were present in the blood samples of both groups. Among these, the expression levels of 12 DEmiRNAs were higher in the low-grade group compared with the high-grade group, whereas the other 15 DEmiRNAs were more highly expressed in the high-grade group compared with the low-grade group $(\mathrm{P}<0.05$; Table VII).

\section{Discussion}

SCG is a tumor of the central nervous system with a relatively low incidence, accounting for $10 \%$ of all types of tumor of the entire spinal canal, with an annual incidence of $\sim 0.22 / 100,000$ in the USA (12). SCG occurrence and development are thought to be the result of the combined influence of genetic and environmental factors that are regulated by multiple genes. With 


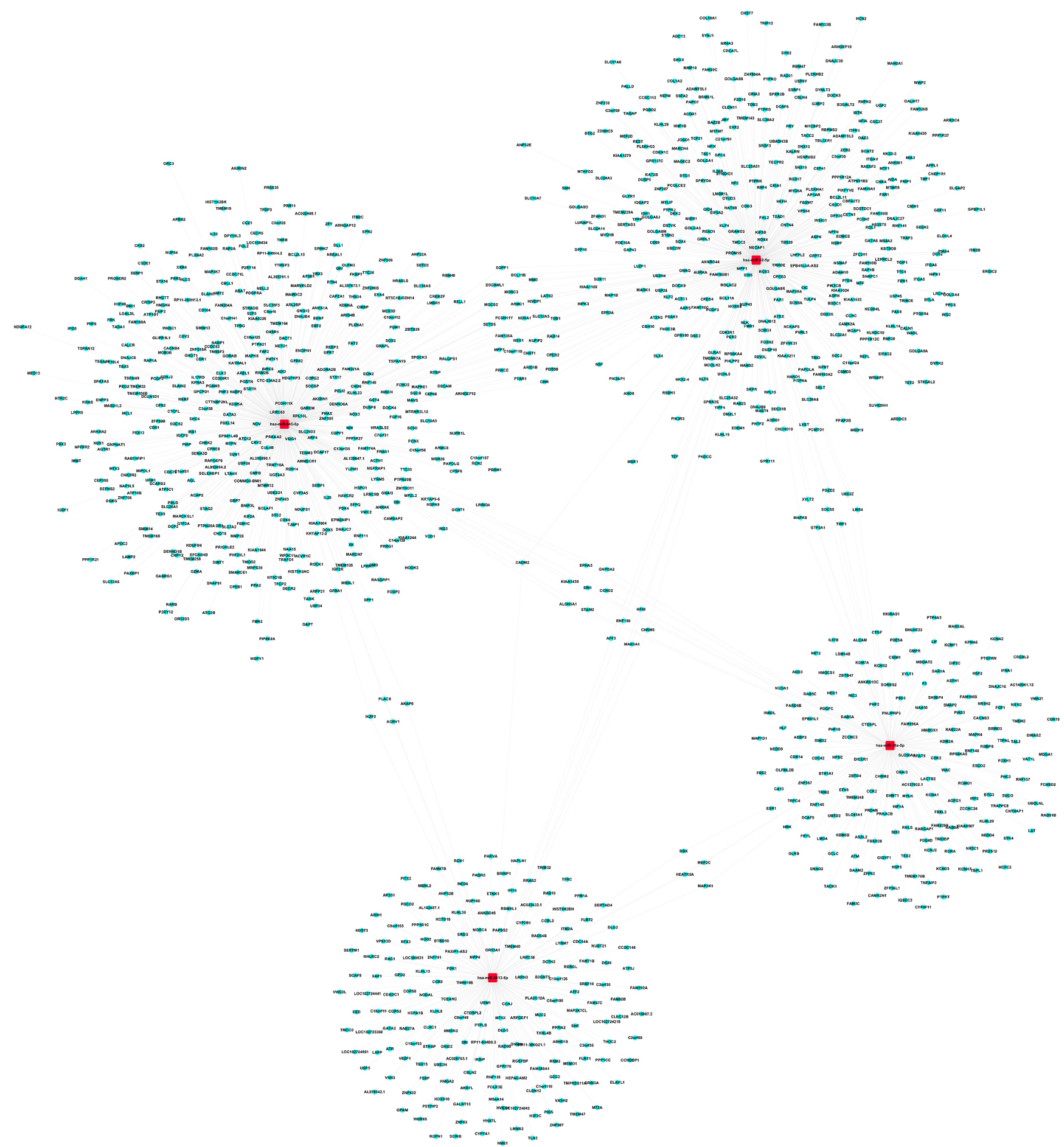

Figure 4. Differentially expressed miRNA-mRNA network of hsa-miR-18a-5p, hsa-miR-545-5p, hsa-miR-3613-5p and hsa-miR-32-5p in patients with high-grade spinal cord glioblastoma. The square, red nodes and the round, blue nodes represent the downregulated miRNAs and mRNAs, respectively, and edges between two nodes represent a potential link. miR, microRNA.

developments in the fields of tumor molecular biology and molecular genetics, multiple molecular changes, including abnormal expression and mutations in miRNAs, have been discovered $(13,14)$. These genetic changes may improve early diagnosis of cancer and typing and prognosis of patients. Therefore, the search for novel, sensitive and specific biomarkers associated with development of SCG are important for early diagnosis, prognostic evaluation and clinical treatment.
At present, a number of studies have focused on the role and abnormal expression of miRNAs in the progression of glioma, but there are fewer studies investigating specific miRNA expression profiles $(15,16)$. The expression of miRNAs in peripheral blood has been associated with different types of tumor and tissue specificity, which has allowed them to be used as a diagnostic criterion (17). In the present study, miRNA microarray technology was used to compare the 
A

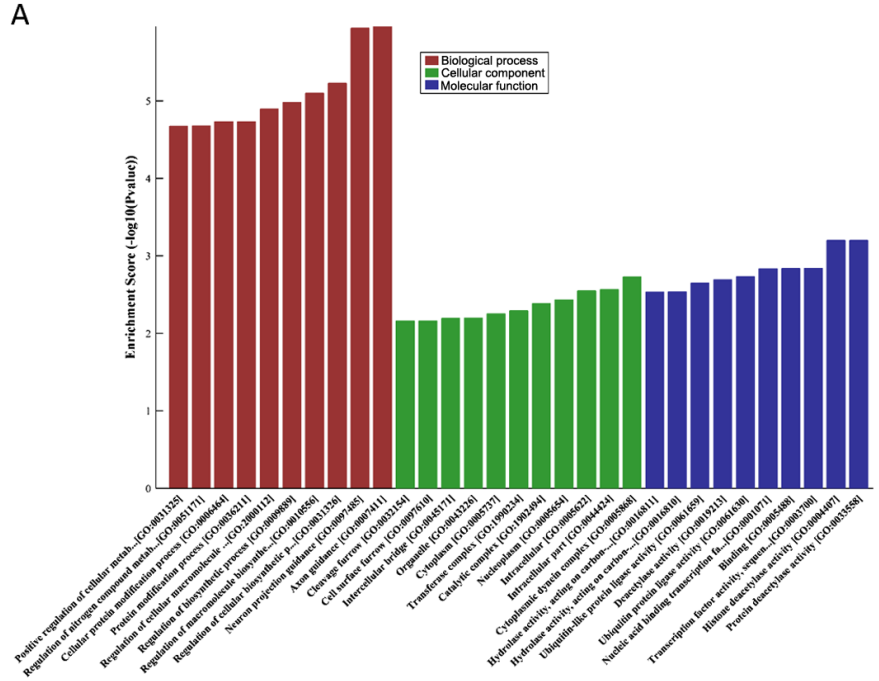

B

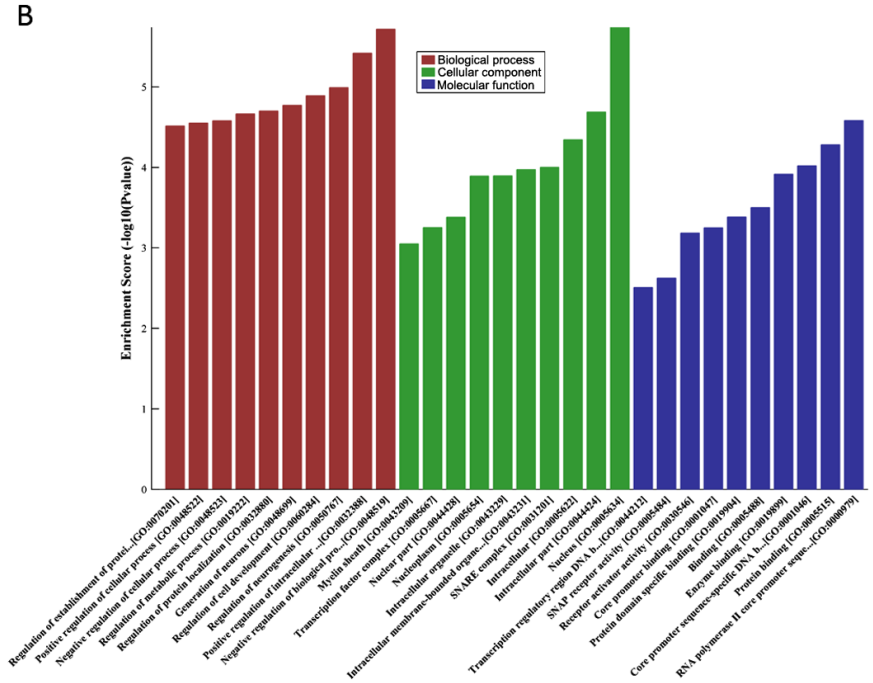

Figure 5. Top 10 enriched GO terms for the DEmiRNAs in the serum samples of patients with low-grade spinal cord glioblastoma. (A) Upregulated and (B) downregulated DEmiRNAs. The abscissa represents the GO term, from left to right, for the biological process, cellular component and molecular function. The ordinate indicates the GO term. GO, Gene Ontology; miRNA, microRNA; DEmiRNAs, differentially expressed miRNAs.

A

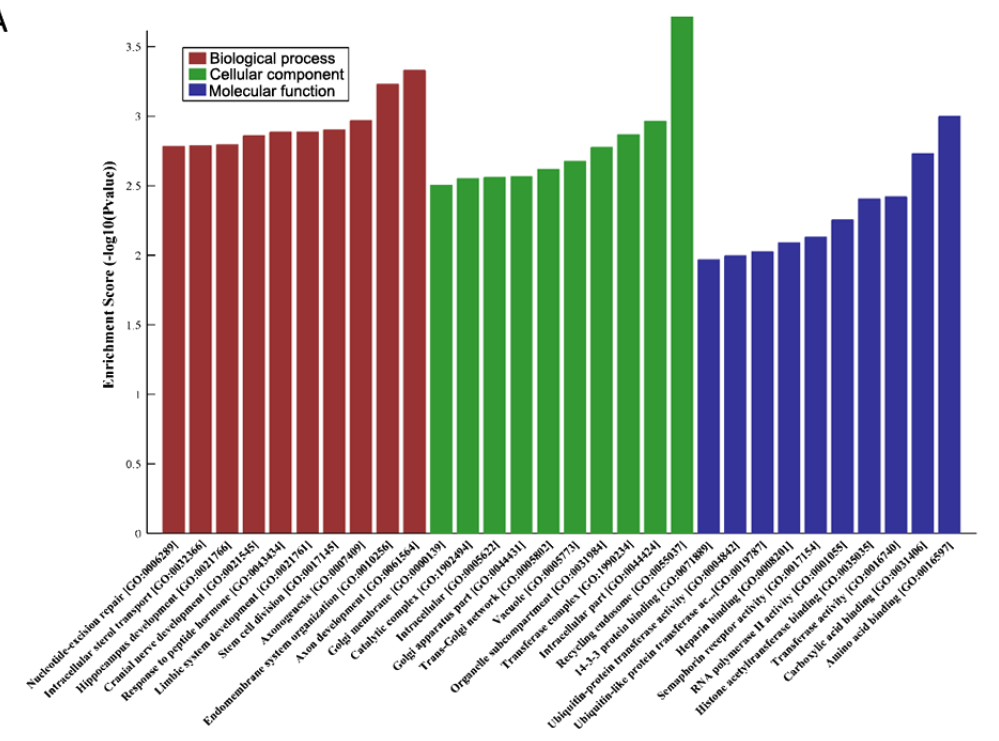

B

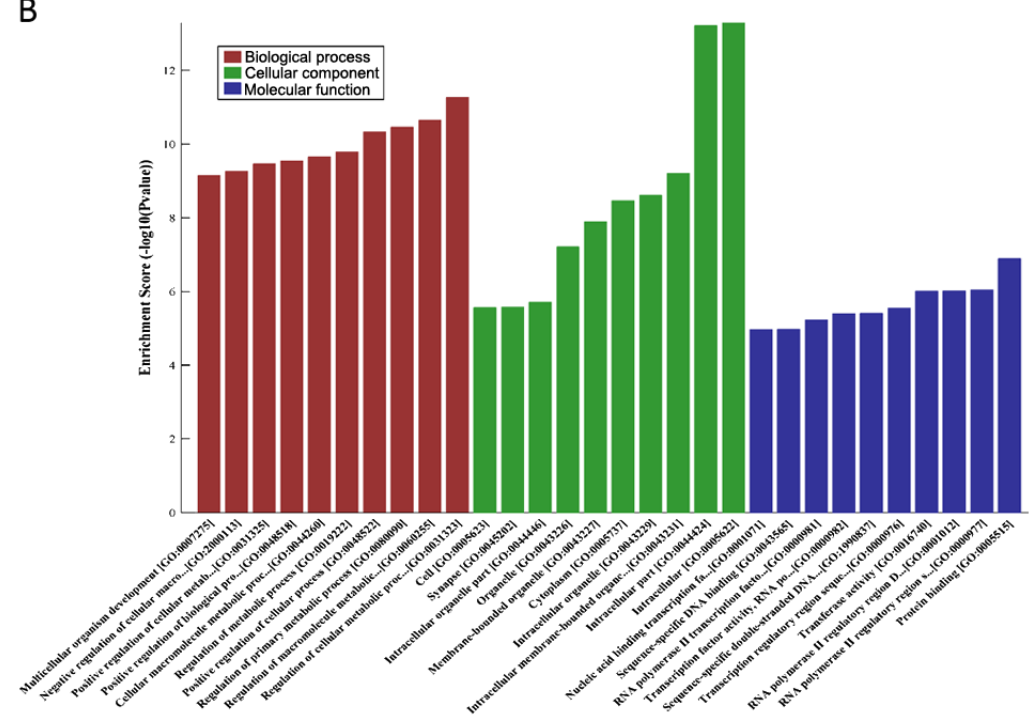

Figure 6. Top 10 enriched GO terms for the DEmiRNAs in the serum samples of patients with high-grade spinal cord glioblastoma. (A) Upregulated and (B) Downregulated DEmiRNAs. The abscissa represents the GO term, from left to right, for the biological process, cellular component and molecular function. The ordinate indicates the GO term. GO, Gene Ontology; miRNA, microRNA; DEmiRNAs, differentially expressed miRNAs. 
A

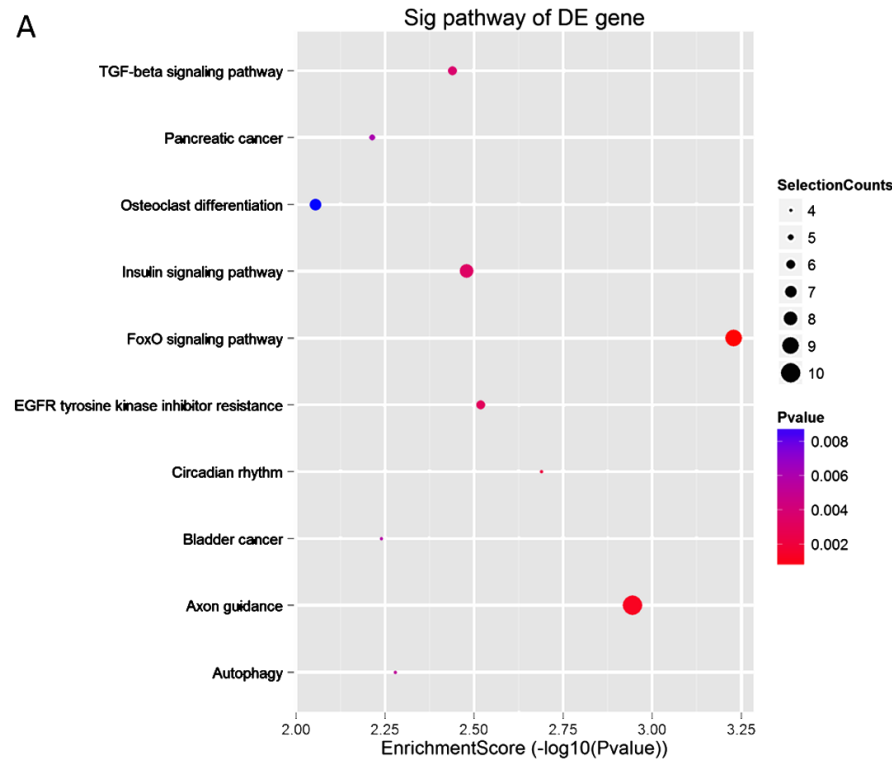

C

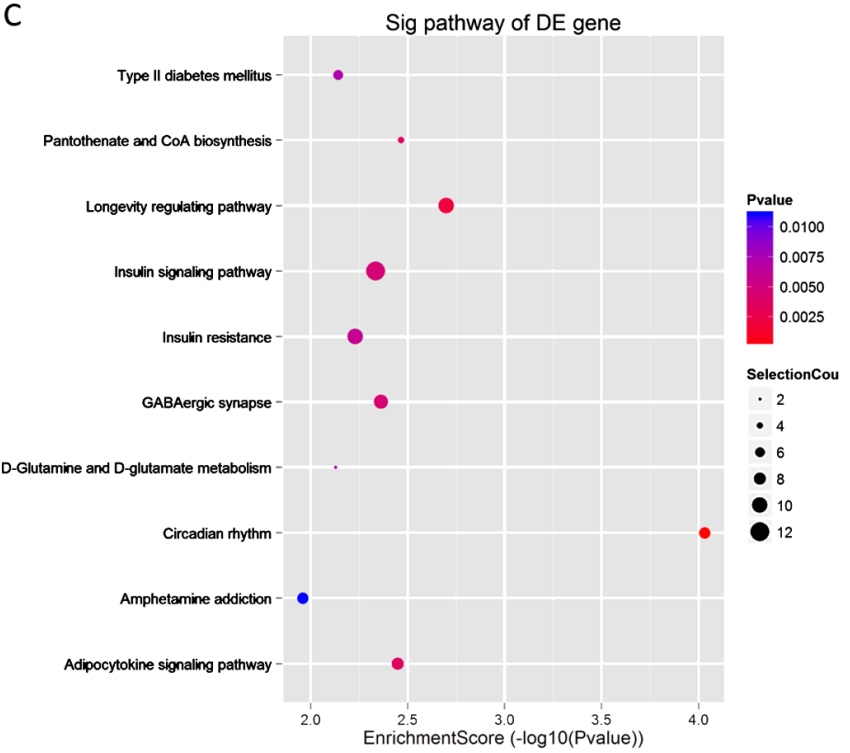

B
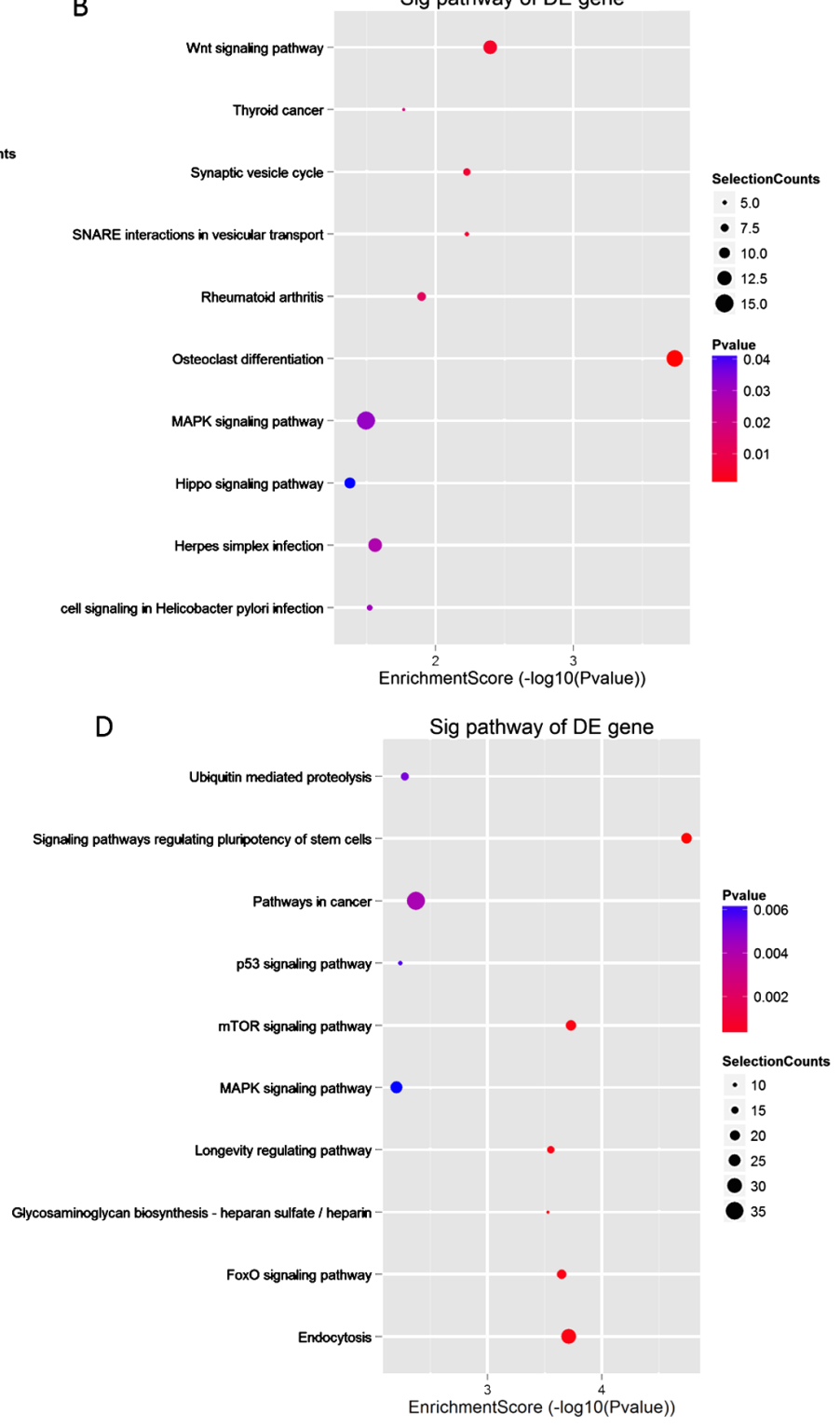

Figure 7. Top 10 enriched pathways based on Kyoto Encyclopedia of Genes and Genomes pathway enrichment analysis of the DEmiRNAs in SCG blood samples. Red represents upregulated miRNAs, while blue represents downregulated miRNAs and the size of the circle corresponds to the number of miRNAs. (A) Signaling pathways associated with upregulated DEmiRNAs in patients with low-grade SCG. (B) Downregulated DEmiRNAs in patients with low-grade SCG. (C) Upregulated DEmiRNAs in patients with high-grade SCG. (D) Downregulated DEmiRNAs in patients with high-grade SCG. miRNA, microRNA DE, differentially expressed; SCG, spinal cord glioma.

expression of miRNAs in the blood of patients with SCG with patients with a benign tumor. The miRNA expression profiles for the different grades of SCG were screened to identify differentially expressed miRNAs which may serve a role in the development of SCG, and thus may serve as biomarkers for specific stages of SCG.

In the present study, 43 and 90 miRNAs were abnormally expressed in the low-grade and high-grade groups, respectively, compared with the matched control group. Certain miRNAs were not abnormally expressed in the low-grade group, but were abnormally expressed in the high-grade group, and these miRNAs may be closely associated with malignant progression of SCG and may serve as useful biomarkers to aid in diagnosis and prognosis of patients. Additionally, whilst the majority of the abnormally expressed miRNAs identified in the low-grade and high-grade groups were different,
27 DEmiRNAs were observed in both groups. Therefore, it is hypothesized that these miRNAs may be involved in the pathogenesis of SCG and their specific mechanism of action will be the focus of future studies.

As an mRNA may be regulated by multiple miRNAs, and one miRNA may regulate multiple mRNAs (18), differentially expressed miRNAs from the low-grade and high-grade groups were selected to construct the miRNA-mRNA networks, enabling further investigation of potential associations at the mRNA and protein levels. Pathway knowledge can provide disease marker information crucial for diagnosis, drug choice and patient treatment (19). The GO and KEGG analysis of the target genes revealed that the target genes of these miRNAs were enriched in processes and signaling pathways associated with the nervous system and tumors. KEGG signaling pathway analysis revealed a number of target genes enriched 
Table VII. Co-expression of differentially expressed miRNAs between blood samples from patients with low-grade or high-grade spinal glioma.

\begin{tabular}{llcr}
\hline Patient ID & \multicolumn{1}{c}{ miRNA } & Low grade, FC & High grade, FC \\
\hline 42530 & hsa-let-7a-2-3p & 3.250 .967 & 2.010 .692 \\
42551 & hsa-miR-122-3p & 5.070 .068 & 6.068 .409 \\
46633 & hsa-miR-1267 & 2.778 .844 & 3.180 .956 \\
46363 & hsa-miR-1272 & 2.277 .376 & 2.086 .202 \\
148536 & hsa-miR-1-5p & 2.235 .554 & 3.068 .592 \\
146165 & hsa-miR-1973 & 2.203 .796 & 2.687 .838 \\
148338 & hsa-miR-3064-3p & 3.556 .303 & 3.537 .826 \\
147651 & hsa-miR-3123 & 4.102 .231 & 3.878 .089 \\
11058 & hsa-miR-325 & 4.038 .391 & 2.888 .924 \\
11083 & hsa-miR-371a-3p & 2.065 .478 & 3.602 .984 \\
168768 & hsa-miR-4423-5p & 22.859 & 2.520 .848 \\
169029 & hsa-miR-4471 & 1.165 .354 & 6.816 .256 \\
169247 & hsa-miR-4477a & 8.457 .066 & 3.624 .097 \\
168801 & hsa-miR-4477b & 8.578 .137 & 3.521 .554 \\
46808 & hsa-miR-4485-3p & 1.477 .512 & 4.332 .511 \\
169369 & hsa-miR-4490 & 5.428 .902 & 418.754 \\
168917 & hsa-miR-4511 & 214.971 & 2.591 .108 \\
29379 & hsa-miR-452-5p & 2.131 .956 & 3.120 .335 \\
169223 & hsa-miR-4680-3p & 5.799 .037 & 443.464 \\
169335 & hsa-miR-4699-5p & 2.611 .289 & 325.914 \\
168910 & hsa-miR-4735-5p & 2.191 .187 & 2.564 .797 \\
169048 & hsa-miR-4736 & 2.331 .175 & 2.060 .894 \\
168860 & hsa-miR-4766-5p & 4.579 .327 & 5.104 .469 \\
169154 & hsa-miR-4803 & 2.315 .542 & 2.705 .104 \\
168896 & hsa-miR-548ao-3p & 2.169 .132 & 2.038 .673 \\
17626 & hsa-miR-575 & 2.654 .789 & 2.269 .628 \\
33407 & hsa-miR-626 & 3.676 .464 & 3.089 .695 \\
\hline
\end{tabular}

FC, fold change; miR, microRNA.

in tumor-related signaling pathways, such as the FoxO, MAPK and Wnt signaling pathways. Abnormal activation of the Wnt signaling pathway is a ubiquitous change observed in numerous different types of cancer, including gliomas, and it affects the proliferation and invasion of glioma cells $(20,21)$. Abnormalities in the MAPK signaling pathway serves a role in the invasion and metastasis of glioma cells and in the maintenance of biological processes, such as the dryness of glioma stem cells (22-25).

The DEmiRNAs investigated in the present study, some of which have been reported in previous studies, regulate the expression of protein-coding genes and affect several biological functions, serving a role in the occurrence and development of diseases. Studies have shown that miR-1246 may be involved in the development of autoimmune diseases and acts as a transfer messenger that leads to DNA damage by directly inhibiting the ligase 4 gene $(26,27)$. The plasma expression levels of miR-1246 has been used as a diagnostic biomarker for hepatocellular carcinoma $(28,29)$, and miR-1246 regulates the progression of breast and prostate cancer, highlighting the potential of miR-1246 in miRNA-based therapies $(30,31)$. In the present study, the expression levels of hsa-miR-1246 were significantly higher in the low-grade group compared with the control group, demonstrating that hsa-miR-1246 expression levels are dysregulated in SCG and therefore highlighting hsa-miR-1246 as a potential target for treatment of SCG.

Several abnormally expressed miRNA molecules identified in the present study have not been reported in previous studies. In the present study, it was shown that miR-4680 was abnormally expressed in SCG, with high expression levels in both the low-grade and high-grade groups for the first time, to the best of our knowledge. Using TargetScan version 7.1, 137 potential target genes associated with miR-4680 were identified. A total of 348 target genes, based on miR-4680 were associated with gliomas, such as cyclin-dependent kinase 6 (CDK6) which is involved in survival of glioma cells (32). Following small interfering RNA mediated downregulation of CDK6 expression levels, tumor cells were arrested in the G1/S phase and cell proliferation was inhibited (32). These newly discovered and aberrantly expressed miRNAs may provide insight into the mechanisms underlying the development 
of SCG, and their role in SCG progression requires further investigation.

In conclusion, the present study compared DEmiRNAs in low-grade and high-grade groups with the control group and identified several miRNAs which may serve a role in the progression of SCG. These identified miRNAs may serve as novel biomarkers or molecular targets for clinical diagnosis and treatment of patients with SCG.

\section{Acknowledgements}

Not applicable.

\section{Funding}

The present study was funded by The National Natural Science Foundation of China (grant no. NSFC81774171), The Tangshan Science and Technology Innovation Team Training Program (grant nos. 18130219A and 19130201C) and The Collaborative innovation project of Chaoyang District in Beijing (grant no. CYXC1719).

\section{Availability of data and materials}

The datasets used and/or analyzed during the present study are available from the corresponding authors upon reasonable request.

\section{Authors' contributions}

GJJ and TF designed the experiments. TA analyzed and interpreted the data. JL, XQZ, YXW, QSY, YFL and TA performed the experiments. CYC, CL, YQW, MHM and BHL interpreted the data. All authors have read and approved the final manuscript.

\section{Ethics approval and consent to participate}

The present study was approved and supervised by The Ethics Committees of Beijing University of Chinese Medicine and Sanbo Brain Hospital, Capital Medical University (Beijing, China). Written informed consent was obtained from all patients prior to the study start.

\section{Patient consent for publication}

Not applicable.

\section{Competing interests}

The authors declare that they have no competing interests.

\section{References}

1. Louis DN, Perry A, Reifenberger G, von Deimling A Figarella-Branger D, Cavenee WK, Ohgaki H, Wiestler OD, Kleihues P and Ellison DW: The 2016 World Health Organization classification of tumors of the central nervous system: A summary. Acta Neuropathol 131: 803-820, 2016.

2. Appin CL and Brat DJ: Molecular pathways in gliomagenesis and their relevance to neuropathologic diagnosis. Adv Anat Pathol 22: 50-58, 2015.
3. Tanaka S, Louis DN, Curry WT, Batchelor TT and Dietrich J: Diagnostic and therapeutic avenues for glioblastoma: No longer a dead end? Nat Rev Clin Oncol 10: 14, 2013.

4. Kalpathy-Cramer J, Gerstner ER, Emblem KE, Andronesi $\mathrm{O}$ and Rosen B: Advanced magnetic resonance imaging of the physical processes in human glioblastoma. Cancer Res 74: 4622-4637, 2014.

5. Dlouhá D and Hubáček JA: Regulatory RNAs and cardiovascular disease-with a special focus on circulating microRNAs. Physiol Res 66 (Suppl 1): S21-S38, 2017.

6. Gregory RI and Shiekhattar R: MicroRNA biogenesis and cancer. Cancer Res 65: 3509-3512, 2005.

7. Xu HS, Zong HL, Shang M, Ming X, Zhao JP, Ma C and Cao L: MiR-324-5p inhibits proliferation of glioma by target regulation of GLI1. Eur Rev Med Pharmacol Sci 18: 828-832, 2014.

8. Duan R, Han L, Wang Q, Wei J, Chen L, Zhang J, Kang C and Wang L: HOXA13 is a potential GBM diagnostic marker and promotes glioma invasion by activating the Wnt and TGF- $\beta$ pathways. Oncotarget 6: 27778-27793, 2015.

9. Cui R, Guan Y, Sun C, Chen L, Bao Y, Li G, Qiu B, Meng X, Pang C and Wang Y: A tumor-suppressive microRNA, miR-504, inhibits cell proliferation and promotes apoptosis by targeting FOXP1 in human glioma. Cancer Lett 374: 1-11, 2016.

10. Gu Y, Zhang Z, Yin J, Ye J, Song Y, Liu H, Xiong Y, Lu M, Zheng $\mathrm{G}$ and $\mathrm{He} \mathrm{Z}$ : Epigenetic silencing of miR-493 increases the resistance to cisplatin in lung cancer by targeting tongue cancer resistance-related protein 1(TCRP1). J Exp Clin Cancer Res 36: 114, 2017.

11. Mo FF, An T, Zhang ZJ, Liu YF, Liu HX, Pan YY, Miao JN, Zhao DD, Yang XY, Zhang DW, et al: Jiang Tang Xiao Ke Granule Play an anti-diabetic role in diabetic mice pancreatic tissue by regulating the mRNAs and MicroRNAs associated with PI3K-Akt signaling pathway. Front Pharmacol 8: 795, 2017.

12. Milano MT, Johnson MD, Sul J, Mohile NA, Korones DN, Okunieff $\mathrm{P}$ and Walter KA: Primary spinal cord glioma: A surveillance, epidemiology, and end results database study. J Neurooncol 98: 83-92, 2010.

13. Jansen M, Yip S and Louis DN: Molecular pathology in adult gliomas: Diagnostic, prognostic, and predictive markers. Lancet Neurol 9: 717-726, 2010.

14. Riddick $G$ and Fine HA: Integration and analysis of genome-scale data from gliomas. Nat Rev Neurol 7: 439-450, 2011.

15. Schratt GM, Tuebing F, Nigh EA, Kane CG, Sabatini ME, Kiebler $M$ and Greenberg ME: A brain-specific microRNA regulates dendritic spine development. Nature 439: 283-289, 2006.

16. Davis-Dusenbery BN and Hata A: MicroRNA in cancer: The involvement of aberrant MicroRNA biogenesis regulatory pathways. Genes Cancer 1: 1100-1114, 2010.

17. Molina-PineloS,SuárezR,PastorMD,Nogal A,Márquez-MartínE, Martín-Juan J, Carnero A and Paz-Ares L: Association between the miRNA signatures in plasma and bronchoalveolar fluid in respiratory pathologies. Dis Markers 32: 221-230, 2012.

18. Sud N, Zhang H, Pan K, Cheng X, Cui J and Su Q: Aberrant expression of microRNA induced by high-fructose diet: Implications in the pathogenesis of hyperlipidemia and hepatic insulin resistance. J Nutr Biochem 43: 125-131, 2017.

19. Jiang G, Ma Y, An T, Pan Y, Mo F, Zhao D, Liu Y, Miao JN, Gu YJ, Wang Y and Gao SH: Relationships of circular RNA with diabetes and depression. Sci Rep 7: 7285, 2017.

20. Wang K, Wang X, Zou J, Zhang A, Wan Y, Pu P, Song Z, Qian C, Chen Y, Yang S and Wang Y: miR-92b controls glioma proliferation and invasion through regulating Wnt/beta-catenin signaling via Nemo-like kinase. Neuro Oncol 15: 578-588, 2013.

21. Zhang H, Qi Y, Geng D, Shi Y, Wang X, Yu R and Zhou X: Expression profile and clinical significance of Wnt signaling in human gliomas. Oncol Lett 15: 610, 2018.

22. Guo G, Yao W, Zhang Q and Bo Y: Oleanolic acid suppresses migration and invasion of malignant glioma cells by inactivating MAPK/ERK signaling pathway. PLoS One 8: e72079, 2013.

23. Dong L, Qi N, Ge RM, Cao CL, Lan F and Shen L: Overexpression of CD133 promotes the phosphorylation of Erk in U87MG human glioblastoma cells. Neurosci Lett 484: 210-214, 2010.

24. Wang BQ, Yang B, Yang HC, Wang JY, Hu S, Gao YS and Bu XY: MicroRNA-499a decelerates glioma cell proliferation while accelerating apoptosis through the suppression of Notch1 and the MAPK signaling pathway. Brain Res Bull 142: 96-106, 2018.

25. Wang R, Deng D, Shao N, Xu Y, Xue L, Peng Y, Liu Y and Zhi F: Evodiamine activates cellular apoptosis through suppressing $\mathrm{PI} 3 \mathrm{~K} / \mathrm{AKT}$ and activating MAPK in glioma. Onco Targets Ther 11: 1183-1192, 2018. 
26. Ishibe Y,Kusaoi M,Murayama G,Nemoto T,Kon T, Ogasawara M, Kempe K, Yamaji K and Tamura N: Changes in the expression of circulating microRNAs in systemic lupus erythematosus patient blood plasma after passing through a plasma adsorption membrane. Ther Apher Dial 22: 278-289, 2018.

27. Mo LJ, Song M, Huang QH, Guan H, Liu XD, Xie DF, Huang B, Huang RX and Zhou PK: Exosome-packaged miR-1246 contributes to bystander DNA damage by targeting LIG4. Br J Cancer 119: 492-502, 2018.

28. Moshiri F, Salvi A, Gramantieri L, Sangiovanni A, Guerriero P De Petro G, Bassi C, Lupini L, Sattari A, Cheung D, et al: Circulating miR-106b-3p, miR-101-3p and miR-1246 as diagnostic biomarkers of hepatocellular carcinoma. Oncotarget 9 : 15350-15364, 2018.

29. Wang Y, Zhang C, Zhang P, Guo G, Jiang T, Zhao X, Jiang J, Huang $X$, Tong $H$ and Tian Y: Serum exosomal microRNAs combined with alpha-fetoprotein as diagnostic markers of hepatocellular carcinoma. Cancer Med 7: 1670-1679, 2018.
30. Bhagirath D, Yang TL, Bucay N, Sekhon K, Majid S, Shahryari V, Dahiya R, Tanaka Y and Saini S: MicroRNA-1246 is an exosomal biomarker for aggressive prostate cancer. Cancer Res 78: 1833-1844, 2018.

31. Li XJ, Ren ZJ, Tang JH and Yu Q: Exosomal MicroRNA MiR-1246 promotes cell proliferation, invasion and drug resistance by targeting CCNG2 in breast cancer. Cell Physiol Biochem 44: 1741-1748, 2017.

32. Chen SM, Chen HC, Chen SJ, Huang CY, Chen PY, Wu TW, Feng LY, Tsai HC, Lui TN, Hsueh C and Wei KC: MicroRNA-495 inhibits proliferation of glioblastoma multiforme cells by downregulating cyclin-dependent kinase 6. World J Surg Oncol 11: 87, 2013.

(c) (i) $९$ This work is licensed under a Creative Commons Attribution-NonCommercial-NoDerivatives 4.0 International (CC BY-NC-ND 4.0) License. 